\title{
A Remote Monitoring System for Cold Chain Logistics by Means of Social Networks
}

\author{
Jiajin Zhang ${ }^{1}$, Lichang Chen ${ }^{2, *}$, Xiuying Tang ${ }^{2}$ and Quan Gao ${ }^{1}$ \\ ${ }^{1}$ School of Science and Information Engineering, Yunnan Agricultural University, Kunming, Yunan, 650201, China; \\ ${ }^{2}$ School of Mechanic and Electronic Engineering, Yunnan Agricultural University, Kunming, Yunan, 650201, China
}

\begin{abstract}
This work proposes a social networks integrated sensor platform for cold chain logistics to effectively assure agri-food safety and quality. Accordingly, this will enhance consumer confidence and reduce the logistics cost. As a case study, employing WeChat social networks, a remote environment monitoring system inside a vehicle has been developed. Experiment results show that the proposed approach is feasible, cost-effective and flexible for exploiting a social networks-based wireless monitoring system quickly.
\end{abstract}

Keywords: Bluetooth low energy, cold chain logistics, wechat, wireless sensor network.

\section{INTRODUCTION}

With the increasing demand for both agricultural crop quantity and quality, precision agriculture (PA) is known as a solution for effectively resolving the issues of food safety and environmental pollution.

PA employs lots of technologies and infrastructures such as data gathering and management, global positioning system (GPS), wireless sensor networks (WSN), and radio frequency identification (RFID) technologies and so on. Various applications of PA have been proposed and researched widely $[3,6]$. The agri-food cold chain system is one of the important PA applications. And the demand for safety in cold chain system has been increased continuously. Keeping agricultural products and managing them in the appropriate environmental conditions are very important in logistics system.

In this study, we focus on the remote monitoring of agrifood cold chain logistics can effectively as a tool to assist in the assurance of agricultural product safety and quality as well as to achieve consumer confidence and reduce the logistics cost.

Currently, Social Network Service (SNS) have become popular and an important part of everyday life for interaction and communication [1].

The SNS (e.g., Twitter, Facebook and linkedIn) makes it possible for people to communicate and share information easily. And The SNS can be defined as web-based social spaces concentrate on facilitating communication, collaboration and content sharing among people who would like to publish, share and discuss short messages on the Web [2].

WeChat is a mobile phone chat software which launched by Tencent company in China in the early 2011. The user can send speech short letter, video, images and text through the rapidly network. WeChat supports for multiple people talk and chat with good friends in more form which is similar to the SMS, MMS, etc. The software of WeChat itself completely is free, and it will not charge for using any function, produced by the WeChat online traffic fee is charged only by the network operators. WeChat works on iOS, Android, Windows Phone, Symbian and Blackberry devices. By the end of 2013, Wechat achieved more than 300 million registered user accounts in only two years [4].

As a consequence, WeChat is the most popular mobile social networks in China. Additionally, WeChat has a stable open Software Development Kit (SDK) that provides rich possibilities to application developers.

This paper proposes a prototype of WeChat integrated wireless sensor networks system to remote monitoring agrifood in cold chain logistics.

The rest of this paper is organized as follows. Section 2 presents an overview on related works. In Section 3, we introduce our proposed system and describe the implementation of a vehicle environment monitoring system adopting WeChat Platform and a WSN. Some experimental results are shown. Finally, Section 4 concludes the paper and presents the future work.

\section{RELATED WORKS}

Wireless sensor networks has extensively been studied in the past few years. This technology has been widely applied to cold chain domain [5-7].

Ruiz-Garcia et al. explores the potential of wireless sensor technology for monitoring fruit storage and transport conditions. It focuses in particular on ZigBee technology with special regard to two different commercial modules [8].

Pervasive computing and sensor technologies offer great potential for improving the efficiency of the food supply 


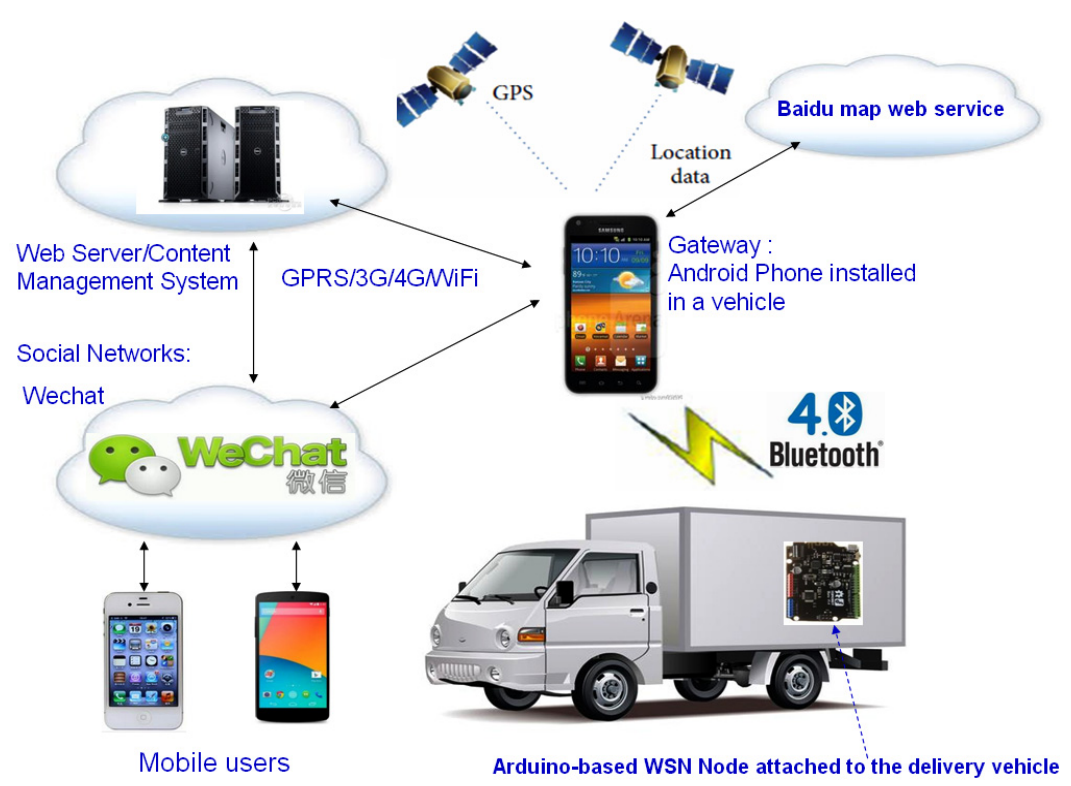

Fig. (1). Architecture of our proposed system.

chain [9]. The paper proposes a telematic platform of an integral nature, enhancing tracking and tracing capabilities for vehicles and goods [10].

In our work, we focus on the ability to locate transport vehicles in cold chain logistics at any time, and allow logistics companies and even consumers to know movements of goods from the source to the destination. Finally, enable logistics companies to assure product quality during transportation.

Simultaneously, some requirements should be considered for the selection of a wireless sensor platform in a cold chain logistics domain:

1) Mobility and scalability, 2) Geolocation support, 3) Ease of software development and hardware design, 4) Costbenefit ratio and 5) Real-time capability.

In view of the above-mentioned requirements, we compare currently offered platforms and propose a new architecture of SNS WeChat integrated wireless sensor networks system to remote monitoring agricultural products in cold chain logistics.

\section{THE DESCRIPTION OF PROPOSED SYSTEM}

\subsection{Architecture of Proposed System with SNS}

Our proposed system is mainly consists of the following elements: (1) environmental monitoring and (2) real-time communication convey sensing data and localization information to remote server and SNS Wechat. In order to monitor the environment inside a transport vehicle, we use the Bluetooth-based sensor node for sensing temperature and humidity. As for real-time data communication and localization, a mobile communication network and GPS functionality of Android smartphone are used. Fig. (1) shows the overall system structure of our proposed system.

In our work, we assume that workflow is as follows. First, Bluno Arduino wireless sensor nodes are able to sample the environment parameters in the interior of a transport vehicle on demand or periodically, and the sensing data are conveyed to a gateway employed an Android smartphone via Bluetooth v4.0 connections. Then, the gateway side application automatically processes the sensing data stored in the local device. The processed result plus the location information obtained through GPS of Android smartphone and Baidu map web service [11] can be further uploaded to a remote Web Server/Content Management System through WiFi or cellular networks (GPRS/3G/4G) and shared with social network WeChat users.

\subsection{Wireless Sensor Node}

Rapid advances in embedded system technologies offer researchers a variety of new and inexpensive solutions for a WSN performs sensing, monitoring, and collecting information.

In addition, the concept of open source hardware, freely provides hardware designs, software programs and development efforts, is accepted widely. In our proposed system, with ease of use, low cost, Low Energy and standardized components and programming language cited as reasons for choosing Bluno Arduino serves as a wireless sensor node.

Bluno Arduino integrates with a TI CC2540 Bluetooth v4.0 chip with the Arduino UNO development board [12]. It allows wireless communication via Bluetooth Low Energy (BLE).

And, for easy interfacing with the sensing and controlling units, including temperature-humidity sensor and light sensor, relay and stepper motor, etc., Bluno Arduino board was used, since Bluno Arduino can provide a rich library for interfacing with the above modules that quickly allows us build high-level WSN's applications.

Meanwhile, in our experiment system, A Bluno Arduino wireless sensor node (Fig. 2) is mainly composed of a Bluno Arduino microcontroller board, a DHT11 is low-cost digital temperature-humidity sensor and a DFRobot BH1750 light intensity sensor (Fig. 3). 


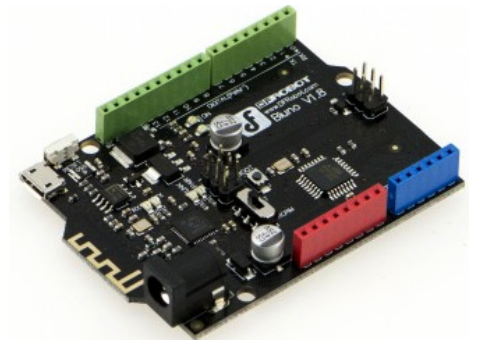

Fig. (2). Bluno arduino board.

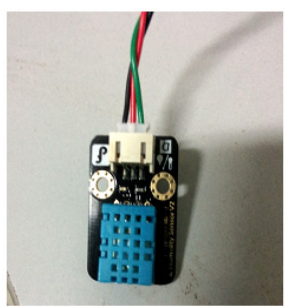

(A)

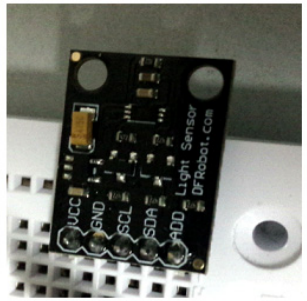

(B)
Fig. (3). (a) Temperature-humidity sensor, and (b) Light intensity sensor.

\subsection{Gateway}

Compared with the traditional and dedicated gateways to bridge sensors and IP networks, Smartphone has significant advantages that make it a promising system platform, which include multiple short/long-distance network interfaces (e.g., WiFi, Bluetooth and cellular network), various integrated sensors, abundant computation and storage resources, and user-friendly interface and development environment.

Moreover, currently the price of Android smartphones has been dropping drastically. These features make it possible to build plenty of embedded sensing and controlling applications based on inexpensive off-the-shelf smartphones.

As a consequence, a powerful mobile computing platform could be used as an alternative gateway of WSN infrastructure, which is ubiquitous and scalable.

In our system, a Samsung Galaxy-S4 Android smartphone that runs Android OS 4.3 on $1.6 \mathrm{GHz} \mathrm{CPU}, 2 \mathrm{~GB}$ DDR2 memory, and Bluetooth v4.0 BLE, is deployed as a gateway, not only for hosting the Internet Proxy, but also for the bridging the gap between SNS WeChat and Bluno Arduino Bluetooth v4.0 sensor nodes in a vehicle.

\subsection{Bluetooth v4.0 Wireless Sensor Network}

In this work, we adopt Bluetooth v4.0 for communications between a gateway Android smartphone and the wireless sensor nodes.

Bluetooth Low Energy (BLE) a feature of Bluetooth v4.0 under the standard IEEE.802.15.1 within a short range (up to 50 meters) can only support peer to peer and star typology, and as a result can not establish a meshed network. the lowenergy feature of a Bluetooth $\mathrm{v} 4.0$ can provide a more power-efficient solution for monitoring applications than existing solutions that use Bluetooth 2.1, ZigBee or WiFi.

Moreover, it has an advantage of being directly compatible with smartphones and tablets, without requiring a dongle or a gateway. This makes possible a new generation of WSN that interact directly with the user.

As seen in Fig. (4), the sensor reading periodically sent by the Bluno Arduino wireless sensor nodes to a gateway through a Bluetooth $\mathrm{v} 4.0$ networks, is composed of a header, ID of wireless sensor node, values measured and checksum.

\subsection{Gateway Side Application}

The wireless sensor nodes send their measurements periodically to a gateway Android smartphone located at a vehicle. Then the information is transmitted to WeChat and remote content management system via the gateway. As a result, a gateway side application is necessary. Fig. (5) shows the relationship among components in our solution.

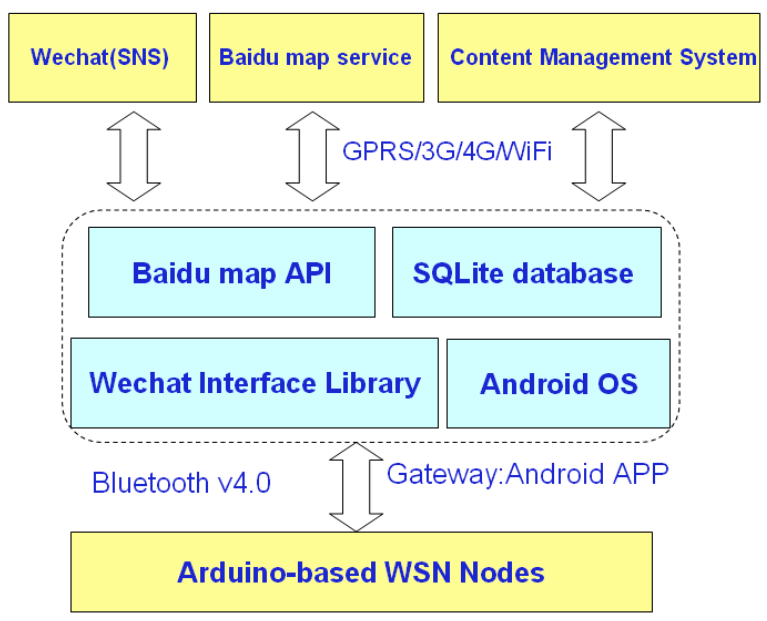

Fig. (5). Block diagram of our proposed system.

As described above, the data from Bluetooth v4.0 wireless sensor nodes is transferred to the gateway side gateway application. then we can obtain the location information of a vehicle through Baidu map web service and a simple location Application Programming Interface (API) contained in Android SDK in our work. Next, all data including sensor data and position information is stored into a SQLite database and processed in order to make text in this application blending with WeChat Android SDK. After that, the application periodically uploads the processed text to WeChat.

The Android SDK of WeChat is a key prerequisite for the realization of the gateway side application consists of three core features including: 1) Log in/out, 2) Take and Renew an access token, and 3) Post and Delete text.

\begin{tabular}{|c|c|l|l|l|l|}
\hline Header & Sensor ID & Value of Temperature & Value of Humidity & Value of Light & Checksum \\
\hline
\end{tabular}

Fig. (4). Format of data package. 
To use the Android SDK to assist the development, developers have to register as a "developer" in WeChat developer open platform and obtain the corresponding App_ID and App_secret to complete the guided registration [4].

Authentication is need before Android SDK works. This SDK provides a WeChat API, when the program is initiated, the following key steps needs to be executed in a gateway side application.

(i) Register to Wechat

// "wx123456" is an APP_ID permitted by Wechat

// IWXAPI is a openapi interface provides the third party $A P P / /$ interact with Wechat

private static final string APP_ID="wx123456"

private IWXAPI api;

private void regToWx () \{

// Through WXAPIFactory, archive a instantiation of IWXAPI

api=WXAPIFactory.creatWXAPI(this,APP_ID,true);

// An APP_ID is registered into Wechat

api.registerAPP(APP_ID);

\}

(ii) Send a request to Wechat

//An object of XTextObject is initiated

WXTextObject textObj = new WXTextObject();

textObj.text $=$ text;

// An object of WXMediaMessage is initiated by WXTextObject

WXMediaMessage msg = new WXMediaMessage();

msg. mediaObject $=$ textObj;

//Send a text message

msg.description = text;

// Construct a Req

SendMessageToWX.Req req = new SendMessageToWX.Req();

//The filed transaction is uniquely identified a request

req.transaction

$=$ string.valueof(system.currenttimeMillis());

req.message $=\mathrm{msg}$;

// Send data to Wechat using api

api.sendReq(req);

(iii) Receive a message from Wechat fest

//Add a WXEntryActivity classin the file Android Mani-

$<$ activity

android:name=".wxapi.WXEntryActivity"

android:lable="@string/app_name"

android:exported="true"/>
After logging into WeChat, the gateway side application is allowed to get an access token by using App_secret. When the expiration of the access token occurs, this application renews the access token every five minutes.

The flow chart of the application is illustrated in Fig. (6). Wireless sensor nodes sample the data every five minutes. After receiving all the data, the application posts the wireless sensor data every five minutes.

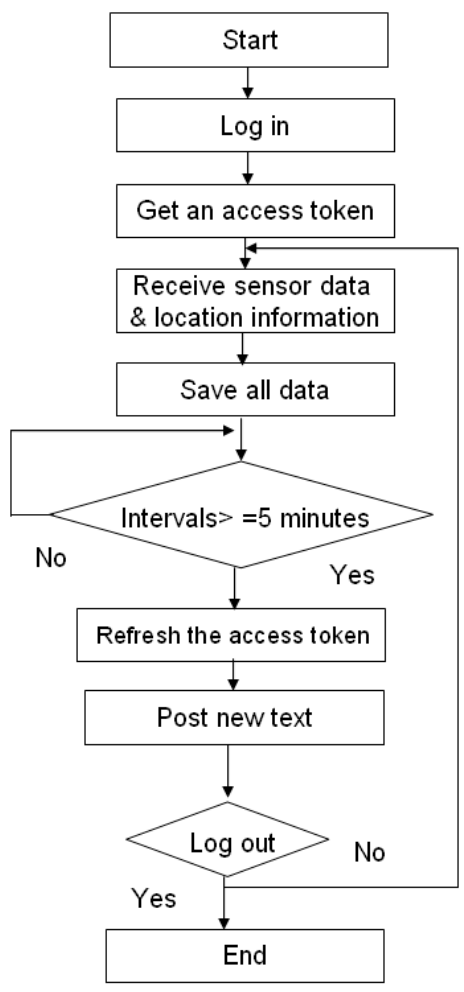

Fig. (6). Flowchart of the gateway side application.

\subsection{Results of the Case Study}

To validate the concept of the proposed system, the developed system is tested by installing the environment condition sensing units and setting up a Bluetooth v4.0 networks in a vehicle, which is deployed as shown in Fig. (7).

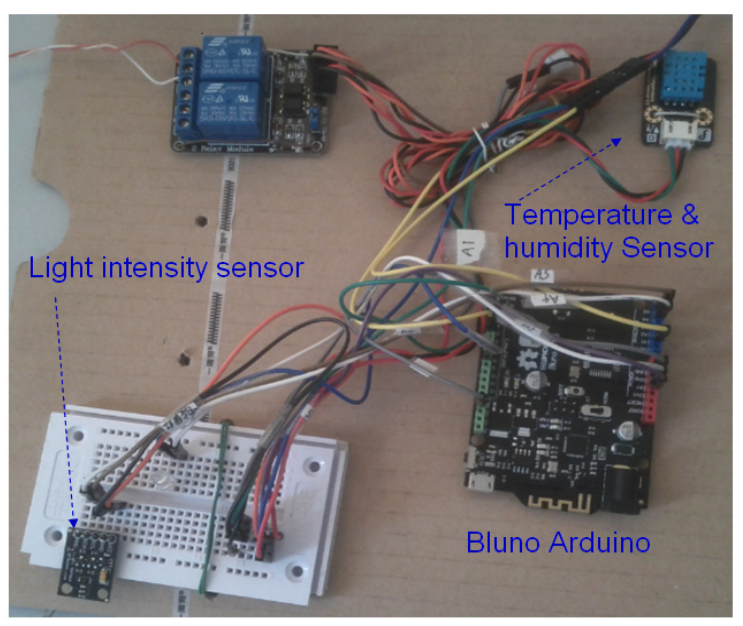

Fig. (7). Testbed of a bluno arduino sensor node in a vehicle. 
Fig. (8) illustrates practical results in a gateway Android smartphone. Here, the conditions in the vehicle were measured at $-8^{\circ} \mathrm{C}, 62 \%$ relative humidity and 36 Lux light intensity.

Time: 14:23:19, July 13,2014
Vehicle ID: YunA521CZ
Air temperature: $-8^{\circ} \mathrm{C}$
Air humidity: $62 \%$
Light intensity: 36 Lux
Location: NO.3 Primary School, Fengyuan Road,
Kunming city, Yunnan Province

Fig. (8). Environment information inside a transport vehicle from a gateway android smartphone.

Fig. (10) depicts periodically posted text information including sensor readings of temperature, humidity and light intensity from a remote transport vehicle are revealed on WeChat.

Vehicle ID: YunA521CZ
Air temperature: $-8^{\circ} \mathrm{C}$
Air humidity: $62 \%$
Light intensity: 36Lux
Location: NO.3 Primary School, Fengyuan Road,
Kunming city, Yunnan Province

Fig. (9). Posted text on wechat.

\subsection{Comparison Between the Previous WSN Solution and our Proposed Approach based on SNS}

Compared with the previous WSN solutions mentioned earlier, there are some advantages in our proposed approach based on SNS. The comparison of two different systems is described as Table $\mathbf{1 .}$

Table 1. Comparison of two different systems.

\begin{tabular}{|c|c|c|}
\hline Items & Our Work & $\begin{array}{c}\text { The Previous } \\
\text { Solutions }\end{array}$ \\
\hline \hline Real-time capability & Good & Fair \\
\hline Mobility & Very good & Fair \\
\hline Openness & Good & Fair \\
\hline Scalability & Very good & Fair \\
\hline Cost-benefit ratio & Excellent & Fair \\
\hline
\end{tabular}

\section{CONCLUSION}

In this paper, by utilizing WeChat open platform, we presented an efficient social network integrated sensor platform for the remote monitoring of agri-food cold chain logistics can effectively as a tool to assist in the assurance of agricultural product safety and quality as well as to achieve consumer confidence and reduce the logistics cost.

In our study, a gateway application running in an Android smartphone for integrating WSN to SNS was developed. In addition, we implemented an environment and position monitoring System in a transport vehicle as a case study.

Our experimental result indicates the feasibility of integrating a WSN monitoring a transport vehicle into the existing infrastructures of the SNS WeChat by presuming upon its open, Web-based SDKs. Our study can lower the implementation cost and complexity, and our proposed solution can provide an alternative common environment for the interaction between SNS and physical world.

In addition, another important feature in our approach has two advantages, namely, simplicity and openness. And we believe that the proposed method can improve the utilization of the cold chain system and its applications.

About the future work, we will intend to incorporate more advanced technology and develop a reliable actuator networks to promptly react to changes of ambient parameters (e.g. temperature, humidity, $\mathrm{CO}_{2}$, light, etc.), and we may improve the applicable areas of the cold chain system.

\section{CONFLICT OF INTEREST}

The authors confirm that this article content has no conflict of interest.

\section{ACKNOWLEDGEMENTS}

This study was supported by an Academic Research Fund of Yunnan Agricultural University in 2015.

\section{REFERENCES}

[1] D. Zeng, S. Guo, and Z. Cheng. The web of things: A survey. Journal of Communications, 6(6):424-438, 2011.

[2] Boyd, D.M., Ellison, N.B. Social network sites: definition, history, and scholarship. J. Comput-Mediat Comm. 13(1), 210-230 (2007).

[3] A. Baggio, "Wireless Sensor Networks in Precision Agriculture," Proc. ACM Workshop Real-World Wireless Sensor Networks, 2005

[4] Wechat open platform.[Online]. Available: https://open.weixin. qq.com.

[5] A. Carullo, S. Corbellini, M. Parvis, and A. Vallan, "A wireless sensor network for cold-chain monitoring," IEEE Trans. Instrum. Meas., vol. 58, no.5, pp.1405-1411, May 2009.

[6] N. Wang, N. Zhang, and M. Wang, "Wireless sensors in agriculture and food industry-recent development and future perspective," Computers and Electronics in Agriculture, vol. 50, no.1, pp. 1-14, 2006

[7] Ruiz-Garcia, L.; Lunadei, L.; Barreiro, P.; Robla, J.I. A review of wireless sensor technologies and applications in agriculture and food industry: State of the art and current trends. Sensors 2009,9,4728-4750.

[8] L.Ruiz-Garcia, P. Barreiro, and J. Robla, "Performance of ZigBeebased wireless sensor nodes for real-time monitoring of fruit logistics," J. Food Eng., vol. 87, no.3, pp.405-415, Aug.2008. 
[9] A. Ilic, T. Staake, E. Fleisch, Using sensor information to reduce the carbon footprint of perishable goods, IEEE Pervasive Computing, 8(1), (2009), 22-29.

[10] J. Santa, M. A. Zamora-Izquierdo, A. J. Jara, and A. F. Skarmeta. Telematic platform for integral management of agricultural/perishable goods in terrestrial logistics. Computers and Electronics in Agriculture, 80:31-40, January 2012.

[11] Baidu location based services open platform. [Online]. Available:http://developer.baidu.com/map

[12] Arduino.[Online]. Available:http://arduino.cc/.

[13] OAuth 2.0.[Online].Available:http://oauth.net/

Received: September 16,2014

Revised: December 23, 2014

Accepted: December 31, 2014

(C) Zhang et al.; Licensee Bentham Open.

This is an open access article licensed under the terms of the Creative Commons Attribution Non-Commercial License (http://creativecommons.org/licenses/by-nc/3.0/) which permits unrestricted, non-commercial use, distribution and reproduction in any medium, provided the work is properly cited. 